\title{
Changes in gonadal gene network by exogenous ligands in temperature- dependent sex determination
}

\author{
Yuiko Matsumoto, Ryohei Yatsu, Caitlin Taylor and David Crews \\ Section of Integrative Biology, University of Texas at Austin, Austin, Texas 78712, USA
}

Correspondence

should be addressed

to $D$ Crews

Email

crews@austin.utexas.edu

\begin{abstract}
We examined the expression of candidate sex-determining genes in the red-eared slider turtle (Trachemys scripta) during the temperature-sensitive period (TSP). Aromatase and Rspo 1 were used as markers of ovarian differentiation and Sox9 was used as a marker of testicular differentiation. Eggs were incubated at a male-producing temperature $\left(26^{\circ} \mathrm{C}\right.$ or MPT) and a female-producing temperature $\left(31^{\circ} \mathrm{C}\right.$ or FPT). First, eggs at the beginning of the TSP (stage 16$)$ were topically treated with the steroid hormones $17 \beta$-estradiol $\left(E_{2}\right)$, testosterone in combination with aromatase inhibitor $(\mathrm{AI}+\mathrm{T})$, the $\mathrm{E}_{2}$ antagonist $(\mathrm{ICI} 182780)$, and the androgen antagonist (flutamide). Secondly, gonads were removed at stage 16 and treated in vitro with $\mathrm{E}_{2}, \mathrm{Al}+\mathrm{T}$, or hormone antagonists. At the $\mathrm{FPT}, \mathrm{Al}+\mathrm{T}$ in ovo suppressed aromatase and Rspo1, while activating Sox9. At the MPT, $E_{2}$ treatment rapidly increased aromatase and Rspo1, while suppressing Sox9. Treatment with the $\mathrm{E}_{2}$ antagonist in ovo decreased aromatase at the FPT. Treatment with the androgen antagonist in ovo increased aromatase and Rspo 1 at early time points at MPT and decreased Sox9 at MPT at later developmental stages. Treatment of isolated gonads cultured in vitro with AI + T at FPT decreased aromatase and Rspo 1 and $\mathrm{E}_{2}$ increased the expression of these genes at MPT. In vitro treatment with $\mathrm{E}_{2}$ antagonist suppressed aromatase and Rspo1 expression at FPT. Overall, our results suggest that exogenous ligands dictate gonadal development by redirecting the expression of candidate sex-determining genes within the genetic cascades induced by temperature.
\end{abstract}

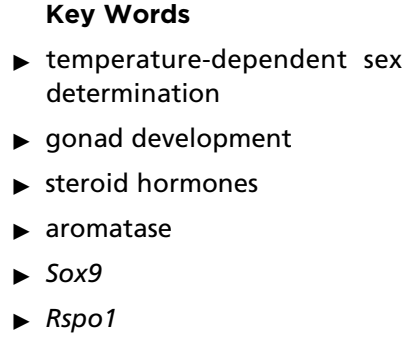

Journal of Molecular Endocrinology (2013) 50, 389-400

\section{Introduction}

Sex determination in vertebrates is a developmental process where the bipotential embryonic gonads differentiate into ovaries or testes depending on external or internal cues. Based on the susceptibility of the embryonic gonads to specific cues, the sex determination system can be divided into two categories: the genotypic sex determination (GSD) system and the environmental sex determination (ESD) system. Temperature-dependent sex determination (TSD) is a form of ESD, where the sex determination of the embryonic gonad is dictated by the incubation temperature of the eggs. In the red-eared slider turtle (Trachemys scripta), a well-studied TSD species, eggs incubated at $26^{\circ} \mathrm{C}$ become males (male-producing temperature or MPT) while eggs incubated at $31^{\circ} \mathrm{C}$ become females (female-producing temperature or FPT). The effect of the incubation temperature is only observed midway through development, also known as the temperature-sensitive period (TSP). Exogenous ligands 
(steroid hormones and steroid metabolism enzyme inhibitors) can override the effect of the incubation temperature if applied during this TSP window. Specifically, $17 \beta$-estradiol $\left(\mathrm{E}_{2}\right)$ treatment of the eggs induces ovarian development at the MPT, while testosterone along with aromatase inhibitor (AI) treatment induces testicular development at the FPT (Wibbels et al. 1991, Crews et al. 1996). Although exogenous ligands can override the effect of the incubation temperature, the role of endogenous sex steroid hormones in sex determination is still somewhat controversial in species with TSD. For example, administration of sex steroid hormone antagonists $-\mathrm{E}_{2}$ antagonist applied at the FPT or androgen antagonist applied at the MPT - often fails to affect the gonad phenotype at hatching (Wibbels et al. 1991, Crews et al. 1996).

How environmental cues, such as temperature or exogenous ligands, engage the molecular mechanisms underlying gonadal development is not fully understood, although recent studies show that several genes (or gene networks) involved in the GSD system exhibit typical gonadal expression patterns in response to temperature in the red-eared slider. For example, aromatase, a gene that encodes the enzyme that catalyzes the conversion of androgens to estrogens, is upregulated at the FPT and downregulated at the MPT in the embryonic gonads (Ramsey et al. 2007). Aromatase expression and activity is not directly involved in sex determination in mammals; however, aromatase is sufficient to control gonadal sex during development in some nonmammalian vertebrates including birds and fish (Elbrecht \& Smith 1992, Guiguen et al. 2010). R-spondin1 (RSPO1), a gene involved in ovarian differentiation in mammals, is also highly expressed during the TSP in red-eared slider gonads at the FPT but is only expressed at low levels at the MPT (Smith et al. 2008). A transcription factor, sex-determining region on Y chromosome-box 9 (Sox9), a direct target of the transcription factor SRY during testicular differentiation in mammals, is upregulated at the MPT and downregulated at the FPT in embryonic gonads (Shoemaker et al. 2007a). Accumulating evidences suggest that Rspo1 and Sox9 are balancing factors to determine a unidirectional result of gonad phenotype in mammals (Huang et al. 1999, Barrionuevo et al. 2006, Parma et al. 2006, Chassot et al. 2008, Tomizuka et al. 2008). In this study, we examined the expression pattern of three candidate sex-determining genes in TSD, aromatase, Rspo1, and Sox9, in exogenous ligand-mediated sex determination in red-eared slider gonads. Slider eggs were topically treated with exogenous ligands at a corresponding temperature at stage 16 , the beginning of the TSP.
As previous studies of the red-eared slider show that treatment with aromatase inhibitor or testosterone alone during development produces limited or no male hatchlings (Wibbels et al. 1991, Crews \& Bergeron 1994, Crews 1996), we used combined treatment with aromatase inhibitor and testosterone at the FPT to ensure mostly male hatchlings in the current study. The expression patterns were then examined during the following developmental time periods including hatching. Furthermore, we examined the effect of hormone antagonists on candidate sex-determining gene expression to understand the role of endogenous sex steroid hormones in gonadal determination. The effect of exogenous ligands and hormone antagonists on candidate sex-determining genes was also examined in isolated cultured gonads.

\section{Materials and methods}

\section{Animals}

Freshly laid red-eared slider turtle eggs were purchased from Clark Turtle Farms (Hammond, LA, USA) and maintained in accordance with humane animal practices under IACUC protocol \# AUP-2011-00149. Eggs were collected daily within $24 \mathrm{~h}$ of laying so that all individual eggs in the shipment were roughly of equal developmental stages. Eggs were mixed, so clutches were equally represented in each experimental group. Eggs were stored at room temperature for 10 days, at which time all eggs were candled to assess viability. Viable eggs were randomly placed in trays with moistened vermiculite and incubated at either $26^{\circ} \mathrm{C}$ (MPT) or $31^{\circ} \mathrm{C}(\mathrm{FPT})$. Incubator temperatures were checked daily with thermometers, and temperature fluctuations were monitored with HOBO data loggers (Onset Computer Corp., Bourne, MA, USA). Embryos were allowed to develop until stage 16, the early TSP when all embryos are sensitive to temperature and exogenous ligands (Wibbels et al. 1991). Staging was according to external morphological characteristics according to the Greenbaum's staging series (Greenbaum 2002).

\section{In ovo exogenous ligand treatment}

At stage 16, eggs were treated as follows, after which the eggs were returned to their designated incubation temperature; at the FPT, eggs were treated with i) $10 \mu \mathrm{ltOH}$ as a vehicle control, ii) $100 \mu \mathrm{g}$ testosterone (Sigma) combined with $75 \mu \mathrm{g}$ non-steroid aromatase inhibitor, fadrozole (AI: Sigma), iii) $100 \mu \mathrm{g} \mathrm{E}_{2}$ antagonist

Published by Bioscientifica Ltd. 
ICI 182780 (Sigma), or iv) $500 \mu \mathrm{g}$ ICI 182 780. At the MPT, eggs were treated with i) $10 \mu \mathrm{lEtOH}$ as a vehicle control, ii) $10 \mu \mathrm{g} \mathrm{E}_{2}$ (Sigma), iii) $1 \mathrm{mg}$ androgen antagonist, flutamide (Sigma), or iv) $2.5 \mathrm{mg}$ flutamide. All chemicals were dissolved in $10 \mu \mathrm{l} 100 \% \mathrm{EtOH}$ and topically applied to the surface of the eggs. The dosages used were based on previous studies and on the solubility of the specific agents (Crews \& Bergeron 1994, Wibbels \& Crews 1994, Wu et al. 2009, Barske \& Capel 2010). These eggs were incubated until the embryos reached stages $17,19,21,23,25$, or hatching, at which time gonads were collected to be processed individually ( $n=6-8$ gonads, i.e. three to four individuals/stage/treatment). Gonads from stage 16 embryos incubating at the FPT and MPT were collected before the treatments to determine a basal gene expression level. Gonads were placed individually into tubes containing $800 \mu \mathrm{l}$ Trizol (Life Technologies), vortexed, and stored at $-80^{\circ} \mathrm{C}$ until RNA extraction. Sex was macroscopically diagnosed in hatchling turtles before processing for gene expression (Crews \& Bergeron 1994). All treatments and sex diagnosis were coded and so blind to the investigators.

\section{In vitro exogenous ligand treatment in tissue culture}

Red-eared slider embryonic gonads were isolated and cultured as described previously (Shoemaker-Daly et al. 2010). Briefly, at stage 16 (Day 0), embryonic gonads were dissected under sterile conditions. The isolated gonads were immediately transferred to a floating $0.4 \mu \mathrm{m}$ Millicell membrane (Millipore, Billerica, MA, USA) placed on the surface of a 24-well plate (BD Falcon, Franklin Lakes, NJ, USA) filled with cell culture media composed of Leibovitz's L-15 medium (+ L-glutamine, phenol red-free; Life Technologies) with 10\% charcoal-stripped fetal bovine serum (FBS; Sigma) and 1\% antibiotic/antimycotic (Life Technologies). Isolated gonads from eggs incubated at the FPT were treated with i) $10 \mu \mathrm{M}$ AI combined with $1 \mu \mathrm{M}$ testosterone or ii) $10 \mu \mathrm{M}$ ICI 182780 and allowed to develop at the FPT. Gonads from eggs incubated at the MPT were treated with i) $100 \mathrm{nM} \mathrm{E}_{2}$ or ii) $10 \mu \mathrm{M}$ flutamide and allowed to develop at the MPT. All chemicals were dissolved in EtOH and adjusted to a final concentration of $0.03 \%$. Both incubation temperatures included two negative control conditions: i) vehicle control of $0.03 \%$ EtOH and ii) negative control gonads without any treatment to reinforce the validity of EtOH as a proper negative control for subsequent gene expression analysis. The dosages used were chosen based on previous in vitro studies performed using other cell types (Yano et al. 1995,
Bhattacharyya et al. 2006, Jolly et al. 2006, La Sala et al. 2010, Yilmaz et al. 2011). Culture plates were incubated in a selfcontained growth chamber under sterile condition. On the day of the tissue culture at stage 16 (Day 0), gonads were also extracted and immediately placed in individual tubes containing $800 \mu \mathrm{l}$ Trizol solution, vortexed, and stored at $-80^{\circ} \mathrm{C}$ until RNA extraction (baseline control). The cell culture media were changed every 2 days, and the same concentration of exogenous ligands or controls was added to the fresh media in each well. Gonads were collected in $800 \mu \mathrm{l}$ Trizol on day $1,4,8,12,16$, or 20 , vortexed, and placed at $-80^{\circ} \mathrm{C}$ until RNA extraction.

\section{Total RNA extraction, cDNA synthesis, and quantitative real-time PCR}

Total RNA was extracted with Trizol by following the manufacturer's protocol (Life Technologies). Subsequently, total RNA was treated with DNA-Free Turbo DNase I (Life Technologies) and reverse transcribed into cDNA using the iScript Kit (Bio-Rad). Relative gene expression was quantified using SYBR green (Life Technologies) on the ABI PRISM 7900HT real-time PCR cycler (Life Technologies). Samples were run in triplicate in quantitative real-time PCR (qPCR), and the median values were used for analysis. Relative gene expressions were measured using previously published aromatase, Rspo1, and Sox9 primers specific to sliders and normalized to the gene expression of protein phosphatase 1 (PP1), a housekeeping gene expressed constitutively relative to the genes of interest in slider gonads (Ramsey et al. 2007, Shoemaker et al. 2007a, Smith et al. 2008). The specificity of qPCR was validated by melting curve analysis. The obtained qPCR data were analyzed using the $\triangle C T$ method and the gene expression fold changes between control and treatment groups were calculated by setting the lowest values of the group as onefold. For functional landscape analysis, qPCR data were represented as a percentage maximum in each gene and plotted by Matlab (MathWorks, Natick, MA, USA).

\section{Statistical analysis}

Because of the asymmetric distribution, the nonparametric test was used to analyze all data. Gonad sex ratio and qPCR data were analyzed using $\chi^{2}$ (Fisher's exact) and Wilcoxon rank-sum test respectively by JMP 8.0 (SAS Institute, Cary, NC, USA). All $P$ values acquired by qPCR data are shown in Supplementary Table 1, see section on supplementary data given at the end of this article. Functional landscape

Published by Bioscientifica Ltd. 
analysis was performed using MultiDimBio package in $\mathrm{R}$ (Scarpino et al. 2013). A $P$ value $<0.05$ was considered to be statistically significant.

\section{Results}

Exogenous ligands, not steroid hormone antagonists, can override the incubation temperature on gonad sex ratio at hatching

In the red-eared slider, a single treatment of exogenous ligands at the beginning of TSP, stage 16, is sufficient to override the ambient temperature and produce a specific gonad phenotype (Wibbels et al. 1991, Crews et al. 1996).

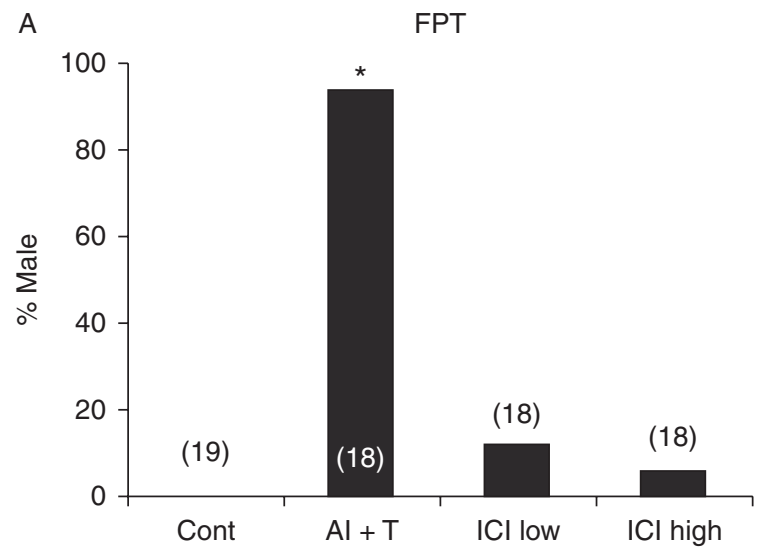

B MPT

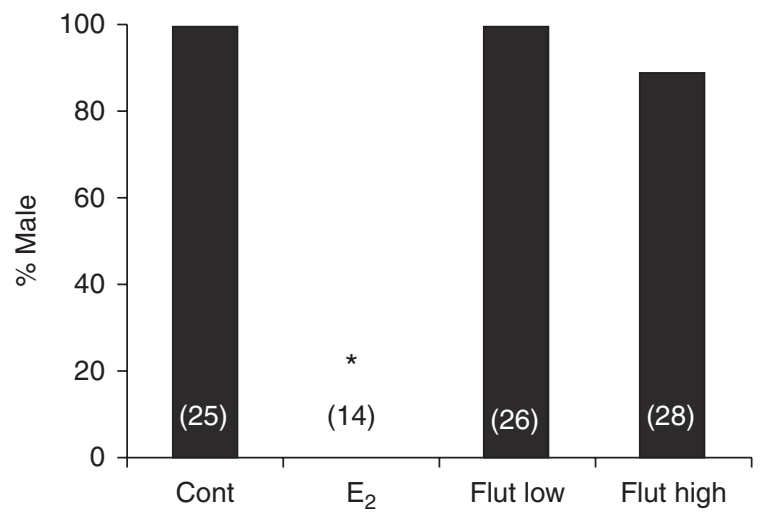

Figure 1

Percentage of male hatchlings in exogenous ligand- and steroid hormone antagonist-treated embryos. Eggs were topically treated with exogenous ligands or hormone antagonists at stage 16, and the gonad phenotype at hatching was examined. (A) FPT and (B) MPT. Values in parentheses indicate the number of individuals. Asterisks indicate statistically significant differences from control groups analyzed by $\chi^{2}$ (Fisher's exact) test. Cont, vehicle control; ICI, $E_{2}$ antagonist ICI 182 780; Flut, androgen antagonist flutamide.
In accordance with this finding, the treatments of exogenous ligands were able to override the temperature effect. $\mathrm{AI}+\mathrm{T}$ at the FPT mostly produced testis phenotypes except one exhibited ovary-like structure with an oviduct (Fig. 1A). Blocking endogenous $\mathrm{E}_{2}$ with a low dosage of ICI 182780 at the FPT produced all ovarian phenotypes except for two eggs that exhibited testes with oviducts; with high doses, all eggs displayed ovarian phenotypes except one that had testes without an oviduct (Fig. 1A). $\mathrm{E}_{2}$ at the MPT produced $100 \%$ ovarian phenotypes at hatching (Fig. 1B). Blocking endogenous testosterone with a low dose of flutamide at the MPT did not have any effect on gonad phenotype, whereas a high dose of flutamide produced three eggs that exhibited ovarian phenotypes with oviduct formation (Fig. 1B). None of the individuals in control groups failed to show the predicted gonad sex according to the ambient temperature. Further, no intersex gonads (e.g. ovotestes) were observed in the treatment groups.

\section{Treatment with testosterone and aromatase inhibitor at FPT reversed the temperature-specific expression patterns of candidate sex-determining genes in ovo}

The expression of candidate sex-determining genes in the control group exhibited a typical temperature-specific expression pattern at FPT: i) an ovarian marker, aromatase exhibited a gradual increase at the beginning of stage 17 , which peaked at stage 21 and gradually decreased untill hatching; ii) another ovarian marker, Rspo1, began to increase after stage 23 and peaked at hatching; and iii) a testicular marker, Sox9, exhibited a low expression throughout development at the FPT (Fig. 2A). These observations are in agreement with previous findings (Ramsey et al. 2007, Shoemaker et al. 2007a,b, Smith et al. 2008), although the current study examines an extended developmental period including hatching. When eggs were topically treated with $\mathrm{AI}+\mathrm{T}$ at stage 16 , the expression of aromatase and Rspo1 decreased as early as stage 17 , and they remained at low levels throughout development (Fig. 2A). The expression of Sox9 in the $\mathrm{AI}+\mathrm{T}$-treated group exhibited a gradual increase throughout development as observed typically at the MPT (Fig. 2A). Next, we performed functional landscape analysis to examine a functional change in the relationship among genes. In this analysis, a transcriptional change in three genes within an individual was considered as one unit, i.e. a functional landscape, and compared between treatments at specific developmental time points (Scarpino et al. 2013). Such analysis will provide us with biologically different aspects of gene functionality

Published by Bioscientifica Ltd 
A
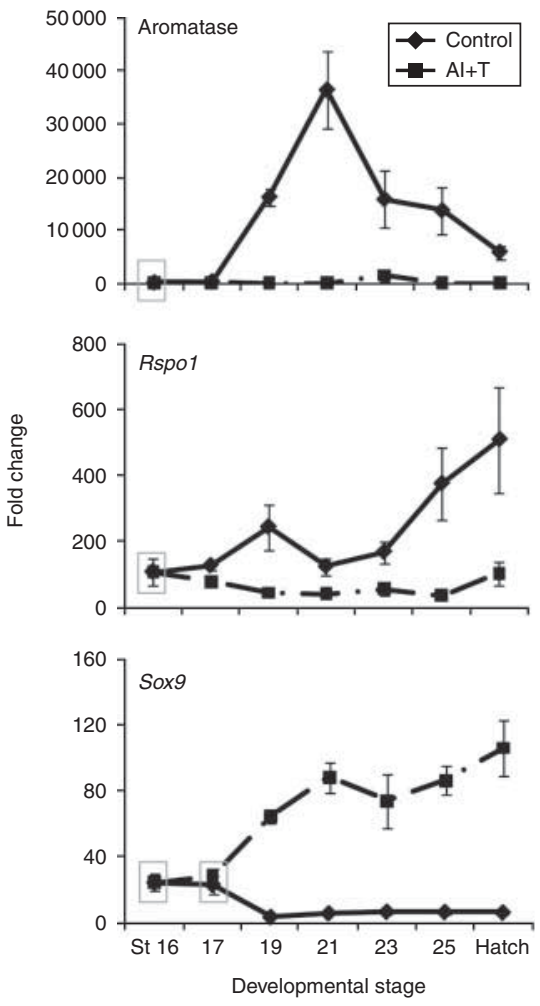

B

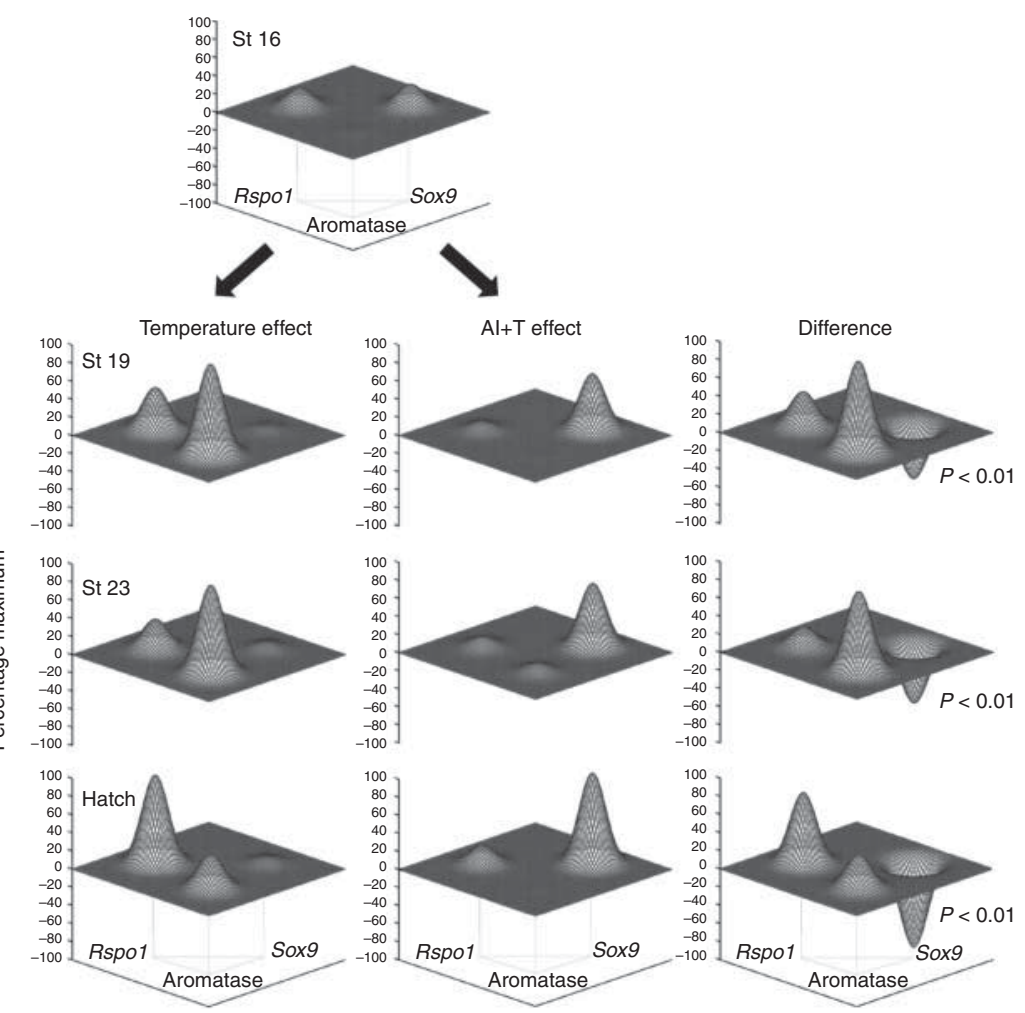

Figure 2

The effect of testosterone and aromatase inhibitor on the expression of aromatase, Rspo1, and Sox9 at the FPT. (A) Changes in gene expression in exogenous ligand-treated slider gonads during development in ovo. Eggs at the FPT were topically treated with $\mathrm{Al}+\mathrm{T}$ at stage 16 . Gonads were collected at stage (St) 17, 19, 21, 23, 25, and hatching. The relative gene expression levels of aromatase, Rspo1, and Sox 9 were measured by qPCR and analyzed using the $\Delta C T$ method. All values were normalized to a housekeeping gene, $P P 1$, and are represented as a fold change. Each time point included $n=6-8$ gonads/treatment/time point. Data represented as

(expression vs interaction), enabling us to follow the 'network' differences in gene expression that covary from one individual to another. The treatment with $\mathrm{AI}+\mathrm{T}$ completely altered the relationship of three gene expressions at the FPT as early as stage 19 (Fig. 2B). Early stage (stage 19) was particularly marked with a presence or absence of prominent aromatase peak followed by a gradual increase in Rspo1 and in Sox9 in control FPT and in $\mathrm{AI}+\mathrm{T}$ treatment group respectively (Fig. 2B).

\section{Treatment with $E_{2}$ at the MPT reversed the temperature-specific expression patterns of candidate sex-determining genes in ovo}

Next, we examined the effect of $E_{2}$ at the MPT on the expression patterns of candidate sex-determining genes, the mean \pm S.E.M. Grey boxes indicate no statistical difference between the two treatments within a developmental stage by Wilcoxon rank sum test. Points without the boxes were significantly different. All $P$ values from statistical analysis are shown in Supplementary Table 1. (B) Landscape analysis for a functional change in the relationship among aromatase, Rspo1, and Sox9. Values from qPCR were represented as a percentage maximum in each gene and plotted by developmental time points. Difference represents a subtraction of a treatment group ( $\mathrm{Al}+\mathrm{T}$ effect) from a control group (temperature effect) in each stage.

aromatase, Rspo1, and Sox9, in red-eared slider gonads. In the control group, aromatase, Rspo1, and Sox9 genes exhibited typical expression patterns found in MPT: suppressed expression of aromatase and Rspo1 and a corresponding increase in the expression of the testicular marker Sox9 (Fig. 3A). The topical addition of $\mathrm{E}_{2}$ at stage 16 reversed the gene expression pattern typically observed at the MPT. The expression of the ovarian marker aromatase increased in response to $\mathrm{E}_{2}$ at stage 23 , which was a later developmental time point and a weaker intensity than the one normally observed at the FPT (Fig. 3A). The expression of Rspo1 was increased as early as stage 17 in rapid response to $\mathrm{E}_{2}$ with an exception of stage 21 (Fig. 3A). Sox9 expression was high at stage 17 in the $\mathrm{E}_{2}$-treated group; however, it decreased throughout the rest of the developmental stages (Fig. 3A). Functional landscape analysis 

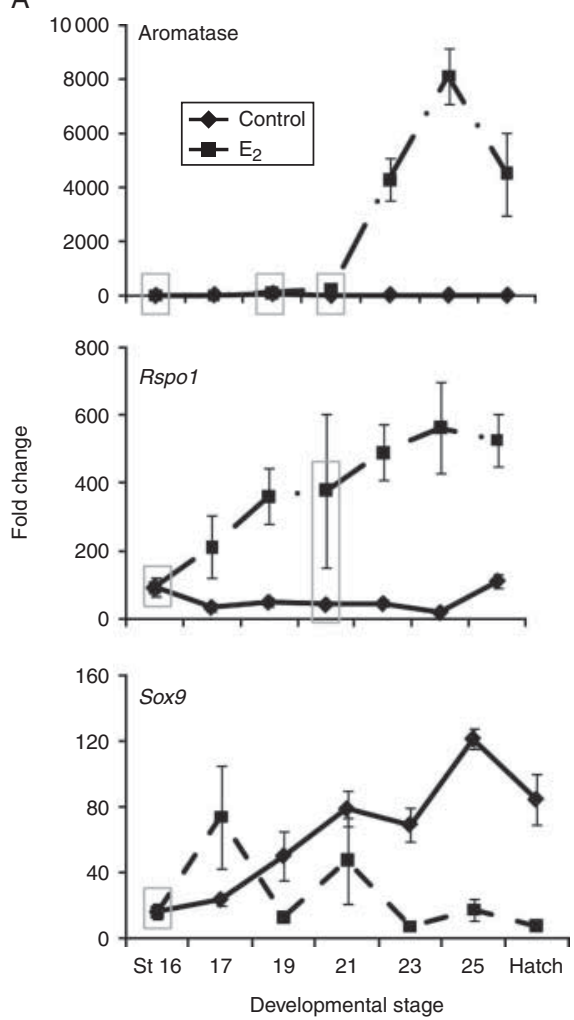

B

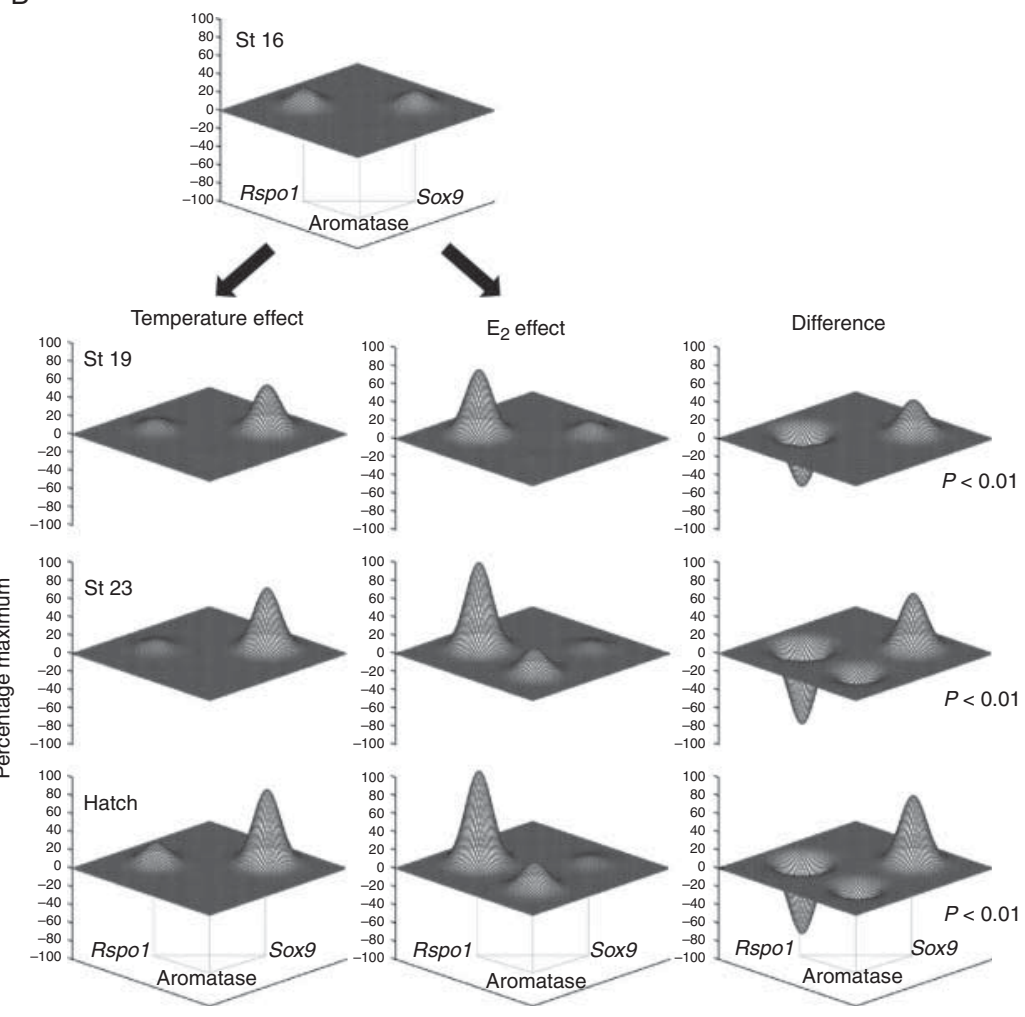

Figure 3

The effect of $E_{2}$ on the expression of aromatase, Rspo1, and Sox9. (A) Changes in gene expression in $E_{2}$-treated slider gonads during development in ovo. Eggs at the MPT were topically treated with $E_{2}$ at stage 16. Gonads were collected at stage (St) 17, 19, 21, 23, 25, and hatching. The relative gene expression levels of aromatase, Rspo1, and Sox9 were measured by qPCR and analyzed using the $\Delta C T$ method. All values were normalized to a housekeeping gene, $P P 1$, and represented as a fold change. Each time point included $n=6-8$ gonads/treatment/time point. Data are represented as the mean \pm S.E.M. Grey boxes indicate no

revealed that a functional relationship of three genes at the MPT was completely altered by a treatment with $\mathrm{E}_{2}$ from the early developmental stages. In $\mathrm{E}_{2}$-treated gonads, Rspo1 expression gradually increased while Sox9 expression was suppressed, as typically observed at the FPT (Fig. 3B). However, unlike a typical landscape at the FPT, it occurred without a spike of aromatase expression (Figs 3B compared to 2B).

\section{Exogenous ligands shape ovary, not testis, phenotype differently from incubation temperature}

We further examined whether a certain phenotype shaped by exogenous ligands followed the same landscape pathway as the phenotype induced by temperature. Eggs incubated at the FPT and those treated with $\mathrm{E}_{2}$ at the MPT produce an ovarian phenotype at hatching statistical difference between the two treatments within a developmental stage by Wilcoxon rank sum test. Points without the boxes were significantly different. All $P$ values from statistical analysis are shown in Supplementary Table 1. (B) Landscape analysis for a functional change in relationship among aromatase, Rspo1, and Sox9. Values from qPCR were represented as a percentage maximum in each gene and plotted by developmental stages. Difference represents a subtraction of a treatment group ( $E_{2}$ effect) from a control group (temperature effect) in each stage.

(Fig. 1). A functional relationship of three candidate sexdetermining genes showed no difference between the FPT and the MPT at stage 16 (before the treatment; Fig. 4A). However, the topical treatment of $E_{2}$ at the MPT at stage 16 had induced a different landscape pattern from control FPT at the midway of development, stages 19 and 23 (Fig. 4A). The difference in landscape pathway diminished at the hatching (Fig. 4A). As opposed to the ovarian phenotype, the landscape pattern of gene expression in testis induced by $\mathrm{AI}+\mathrm{T}$ treatment at the FPT exhibited no difference from the testis induced by control MPT throughout development (Fig. 4B). These results agree with a phenotypic difference between temperature- and ligand-induced gonads, especially prominent in ovarian formation, reported in red-eared slider gonads previously (Barske \& Capel 2010, Matsumoto \& Crews 2012).

Published by Bioscientifica Ltd. 
A
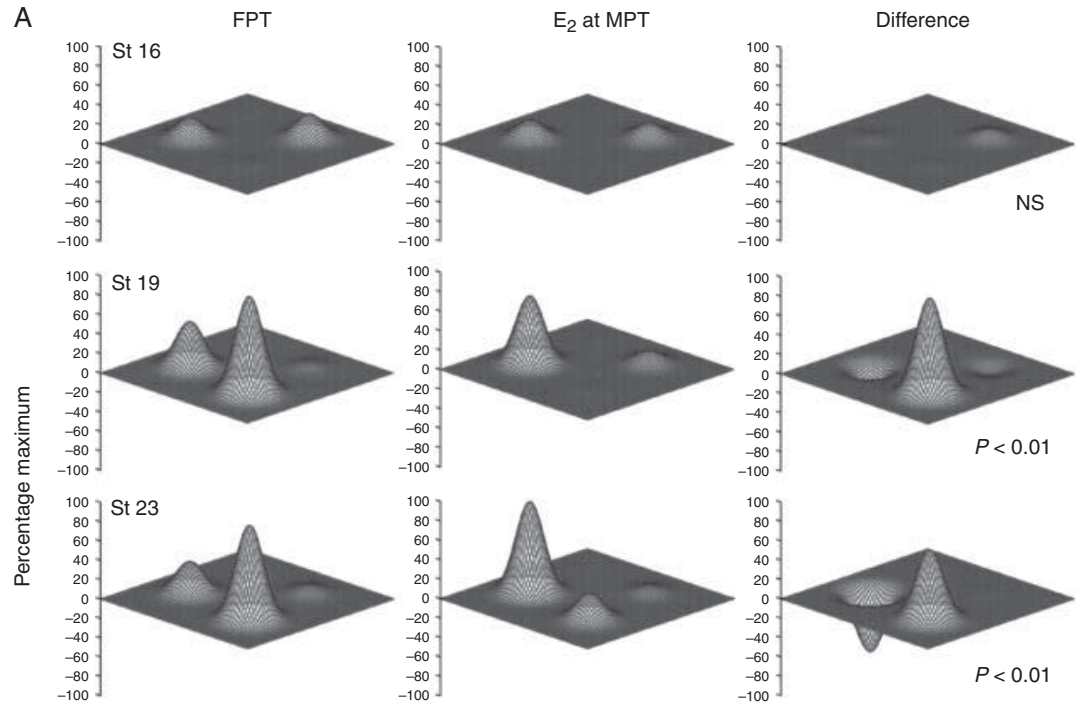

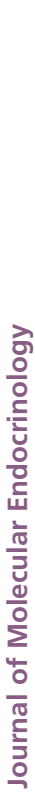
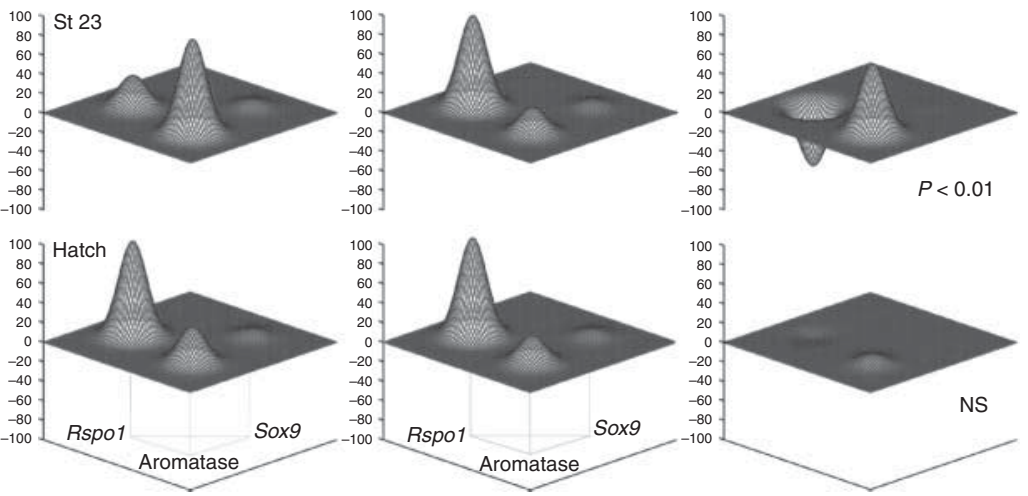

B
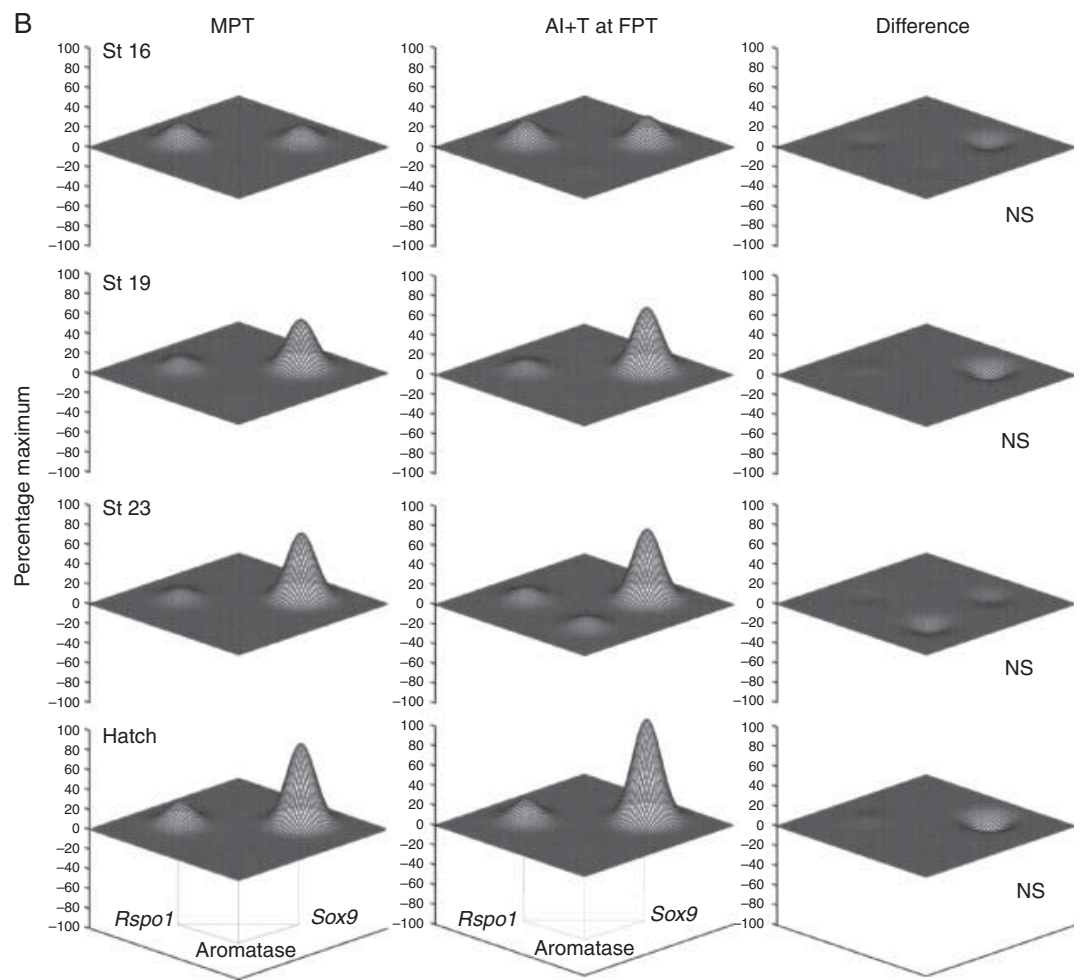

\section{Figure 4}

Landscape analysis compared within a gonad phenotype, ovary (A) and testis (B). The value of qPCR was represented as a percentage maximum in each gene and plotted by developmental stages (St). Difference was calculated by subtraction of a treatment group ( $E_{2}$ at MPT or Al+T at FPT) from a control temperature (FPT or MPT respectively) and statistical differences were analyzed using MultiDimBio package in $\mathrm{R}$ (Scarpino et al. 2013). NS, not statistically significant.

Published by Bioscientifica Ltd. 
Treatment with steroid hormone antagonists altered the temperature-specific expression patterns of candidate sex-determining genes in ovo

Next, we examined the role of endogenous sex steroid hormones in the expression of candidate sex-determining genes at a specific temperature, i.e. a role of $\mathrm{E}_{2}$ to induce ovaries at the FPT or testosterone to induce testis at the MPT. Eggs were topically treated with $\mathrm{E}_{2}$ antagonist at the FPT or testosterone antagonist at the MPT at stage 16 . These treatments did not significantly alter the gonad sex ratio or phenotype from control group at hatching (Fig. 1). However, the expression levels of several genes were affected during development. The addition of low doses of the $\mathrm{E}_{2}$ antagonist ICI 182780 at FPT delayed the peaking of aromatase expression; however, the overall expression pattern of aromatase was similar to the control
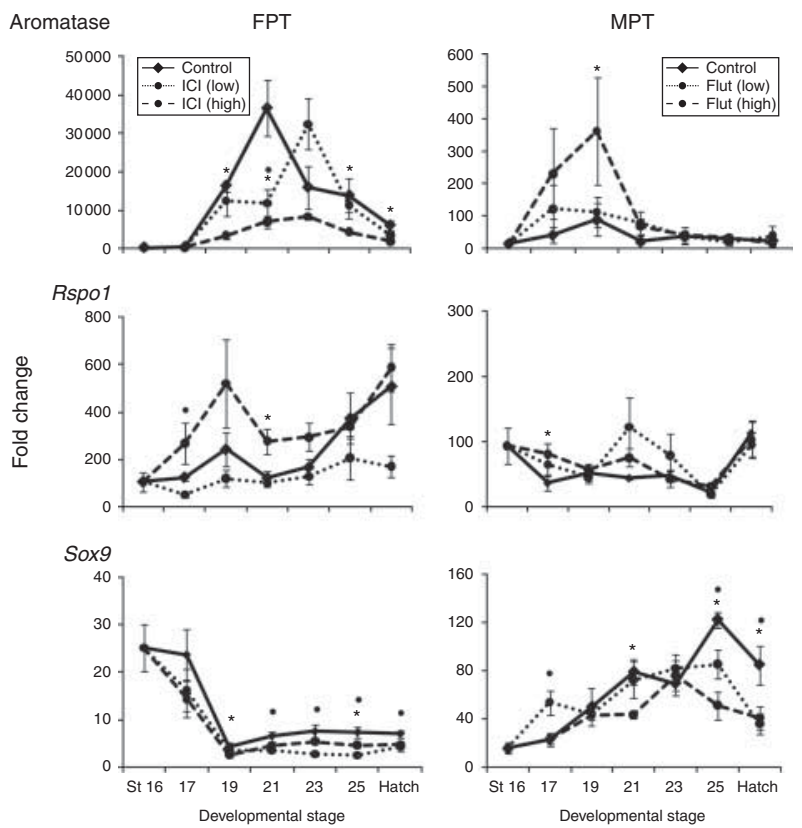

Figure 5

The effect of steroid hormone antagonists on the expression of aromatase, Rspo1, and Sox9 in slider gonads during development. Eggs were topically treated with steroid hormone antagonists ( $E_{2}$ antagonist at FPT and androgen antagonist at MPT) at stage 16 . Gonads were collected at stages $17,19,21,23,25$, and hatching. Relative gene expression levels of aromatase, Rspo1, and Sox9 during development were measured by qPCR and analyzed using the $\Delta C T$ method. All values were normalized to a housekeeping gene, $P P 1$, and represented as a fold change. The same sample was used for the values at stage 16 in both a control and a treatment group. Each time point included $n=6-8$ gonads/treatment/time point. Data are represented as the mean \pm s.E.M. A black circle and an asterisk indicate statistically significant difference(s) in low and high dosages of antagonists respectively from control group analyzed by Wilcoxon rank-sum test. All $P$ values are shown in Supplementary Table 1. $\mathrm{ICI}, \mathrm{E}_{2}$ antagonist ICI 182780 ; Flut, androgen antagonist flutamide. group (FPT in Fig. 5). High doses of ICI 182780 significantly lowered the expression level of aromatase throughout development at the FPT. A low dose of ICI 182780 decreased the expression of Rspo1 at stage 17 at the FPT; however, the expression patterns during the rest of the developmental stages were not altered. In contrast to our prediction, a high dose of ICI 182780 increased Rspo1 expression at stages 17 and 21 at the FPT (FPT in Fig. 5). The expression of Sox 9 significantly decreased with the treatment of low and high doses of ICI 182780 at stages $21,23,25$, and hatching and at stages 19 and 25 at the FPT respectively; the overall expression pattern of Sox 9 during gonadal development, however, remained consistent to control at both doses (FPT in Fig. 5). Next, we examined the role of endogenous testosterone in the expression of candidate sex-determining genes at the MPT by topically applying the androgen antagonist flutamide on the eggs at stage 16. A low dose of the androgen antagonist at MPT did not affect aromatase and Rspo1 expression. With high doses of flutamide at the MPT, there was an increased expression of aromatase at stage 19 and Rspo1 at stage 17, though these effects diminished in subsequent stages (MPT in Fig. 5). The expression of Sox9 was increased at stage 17 and decreased at stage 25 and hatching by low doses of flutamide, while high doses led to decreased expression at stages 21,25 , and hatching (MPT in Fig. 5).

\section{The expression pattern of aromatase and Rspo1 dictated by exogenous ligands and steroid hormone antagonists in vitro mimics the expression pattern observed in ovo}

In previous studies, we showed that the expression pattern of candidate sex-determining genes in isolated, cultured gonads closely mimicked the expression pattern in ovo in response to incubation temperatures; this finding suggests that the gonad itself has the ability to dictate its own gene expression pattern according to environmental cues (Shoemaker-Daly et al. 2010). In this study, we determined whether the observed gene expression profiles elicited by exogenous ligands and hormone antagonists also occurred in isolated gonads. The expression of the testicular marker Sox9 was not included in our data analysis because it showed a significant change in expression by the control vehicle treatment $(0.03 \% \mathrm{EtOH})$ when compared with non-EtOH treatment (data not shown). The expression levels of the two ovarian markers were not affected by the control vehicle treatment and were subsequently analyzed. Treatment with $\mathrm{AI}+\mathrm{T}$ repressed aromatase expression at days 1, 12, and 20 at the FPT in vitro (FPT

Published by Bioscientifica Ltd. 


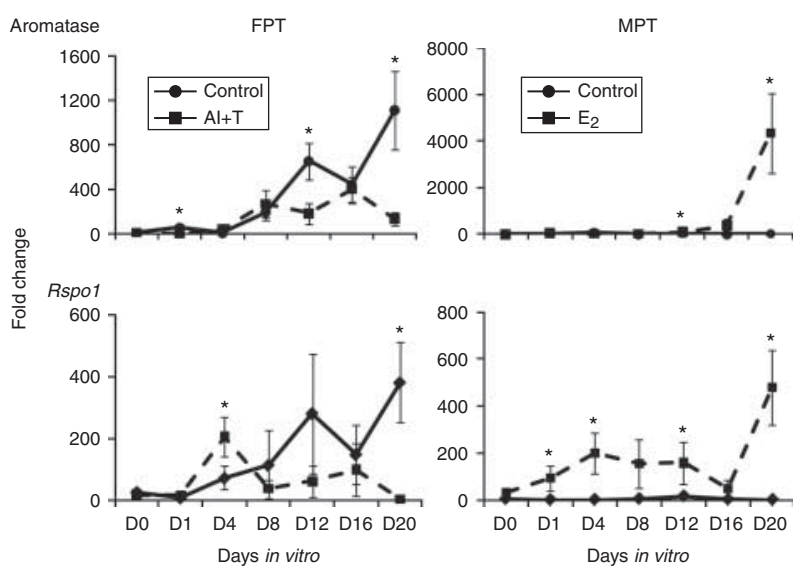

Figure 6

The effect of exogenous ligands on the expression of aromatase and Rspo1 in cultured slider gonads during development. Embryonic gonads at the FPT and MPT were isolated at stage 16, treated with exogenous ligands, and continuously cultured at the same temperature for up to 20 days. The control vehicle group was treated with a final concentration of $0.03 \%$ EtOH. Gonads were collected on days (D) 1, 4, 8, 12, 16, and 20. Relative gene expression levels of aromatase and Rspo1 during development were measured by qPCR and analyzed using the $\triangle C T$ method. All values were normalized to a housekeeping gene, $P P 1$, and represented as a fold change. The same sample was used for the values at stage 16 in both a control and treatment group. Each time point included $n=8-10$ cultured gonads/treatment/time point. Data are represented as the mean \pm s.E.M. An asterisk indicates statistically significant difference (s) from control group analyzed by Wilcoxon rank-sum test. All $P$ values are shown in Supplementary Table 1.

in Fig. 6). The expression of Rspo1 was decreased by $\mathrm{AI}+\mathrm{T}$ treatment at day 20 at the FPT, but its expression was not affected at any other time points (FPT in Fig. 6). $E_{2}$ treatment increased the aromatase expression at days 12 and 20 and Rspo1 expression at days 1, 4, 12, and 20 respectively at the MPT (MPT in Fig. 6). As observed in ovo, the treatment with $\mathrm{E}_{2}$ antagonist ICI 182780 repressed aromatase expression at days 8,12 , and 20 at the FPT in vitro (FPT in Fig. 7). The expression of Rspo1 was also decreased by ICI 182780 treatment at days 8 and 20 at the FPT (FPT in Fig. 7). The androgen antagonist flutamide altered the expression of aromatase at days 1 and 16 and Rspo1 at days 4, 8, and 12 at the MPT (MPT in Fig. 7).

\section{Discussion}

Sex determination in vertebrates takes place as a gradual process and can be categorized into three phases, onset by genetic or environmental triggers, differentiation into testis or ovary, and maintenance by male- or femalespecific molecules. At which phase of the sex determination process the sex steroid hormones come into play remains elusive in nonmammalian vertebrates. However, studies demonstrate that disturbance of sex steroid hormone balance during gonadogenesis often results in redirecting the gonad development against genetic or environmental triggers in nonmammalian vertebrates (Elbrecht \& Smith 1992, Crews \& Bergeron 1994, Kitano et al. 2000). In this study, we investigated the effect of exogenous ligands, i.e. sex steroid hormones and a steroid metabolism enzyme inhibitor, and steroid hormone antagonists on the process of gonad sex determination by examining the patterns of sex-determining gene expression in the red-eared slider turtle. It is known that exogenous ligands can modify the trajectory of gonadal sex determination set by ambient temperature if applied during development in the red-eared slider and other species (Bull \& Vogt 1981, Wibbels et al. 1991, Crews et al. 1996). In the hatchlings with $\mathrm{AI}+\mathrm{T}$ treatment, the typical developmental expression pattern of ovarian markers aromatase and Rspo1 was suppressed and the testicular marker Sox9 was increased (Fig. 2). By contrast, $\mathrm{E}_{2}$
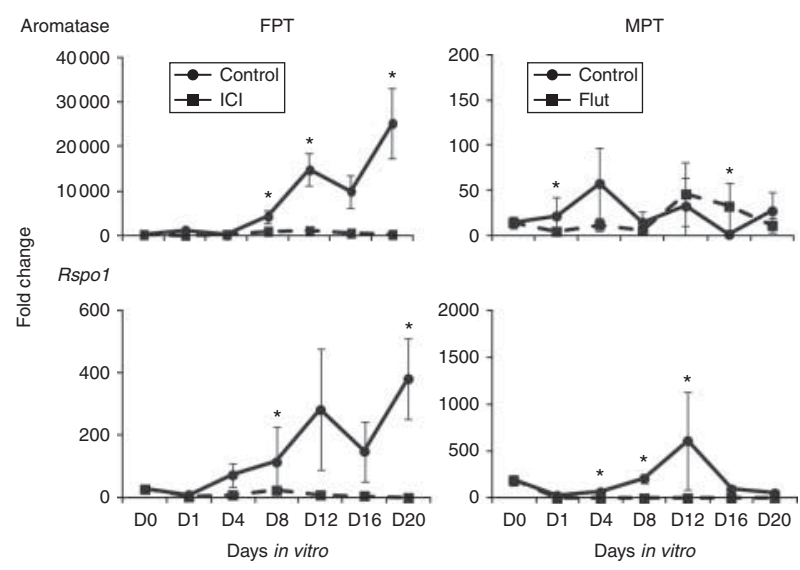

\section{Figure 7}

The effect of steroid hormone antagonists on the expression of aromatase and Rspo 1 in cultured slider gonads during development. Embryonic gonads at the FPT and MPT were isolated at stage 16, treated with steroid hormone antagonists ( $E_{2}$ antagonist at the FPT and androgen antagonist at the MPT), and continuously cultured at the same temperature for up to 20 days. The control vehicle group was treated with a final concentration of $0.03 \%$ EtOH. Gonads were collected on days (D) 1, 4, 8, 12, 16, and 20 . Relative gene expression levels of aromatase and Rspo 1 during development were measured by qPCR and analyzed using the $\Delta C T$ method. All values were normalized to a housekeeping gene, $P P 1$, and represented as a fold change. The same sample was used for the values at stage 16 in both a control and a treatment group. Each time point included $n=8-10$ cultured gonads/treatment/time point. Data are represented as the mean \pm S.E.M. An asterisk indicates statistically significant difference (s) from control group analyzed by Wilcoxon rank-sum test. All $P$ values are shown in Supplementary Table 1. ICI, $E_{2}$ antagonist ICI 182 780; Flut, androgen antagonist flutamide. 
treatment at the MPT during TSP increased aromatase and Rspo1 and decreased Sox9 expression, which resulted in the production of all female hatchlings (Figs 1 and 2). $\mathrm{E}_{2}$-treated gonads at the MPT exhibited a missing peak of aromatase expression while typical Rspo1 and Sox9 expression patterns at the MPT were observed. It suggests that Rspo1 activation is a key signal to initiate ovarian differentiation, and exogenous $\mathrm{E}_{2}$ plays a role in triggering this process when aromatase expression is absent at the MPT. Previous studies also showed that exogenous ligands modify the expression of candidate sex-determining genes, Rspo1 and Sox9. For example, embryonic exposure to DHT increases Sox9 and suppresses Rspo1 mRNA levels in developing mouse prostates (Schaeffer et al. 2008). In the red-eared slider, multiple treatments of eggs with $\mathrm{E}_{2}$ completely suppress the expression of Sox 9 by embryonic stage 19 (Barske \& Capel 2010). Moreover, Sox 9 mRNA is increased in adult ovaries of mice lacking the presence of estrogen receptors (Couse et al. 1999, Dupont et al. 2003). In chickens, the inhibition of estrogen synthesis by aromatase inhibitors results in a decrease in RSPO1 expression in embryonic gonads of genetic females (Smith et al. 2008). Although the hierarchy of genetic cascade of these genes during sex determination has not been established in TSD species, we applied a new analytic method to observe relationship of three genes, a snapshot of genetic interaction at a particular developmental time point. In the functional landscape analysis, the expression pattern of candidate sex-determining genes within an individual is considered as one network or landscape and compared to the networks/landscapes induced by various environmental cues (Scarpino et al. 2013). Interestingly, when the landscape is compared within a phenotype, $\mathrm{E}_{2}$ induced ovarian development exhibits a different developmental pattern of the landscape network from FPTinduced ovarian development (Fig. 4A). This observation confirms that the gonads at the FPT and $\mathrm{E}_{2}$-treated gonads at the MPT follow different gonad differentiation pathways. In testicular development, however, the difference in the gene network between the MPT and $\mathrm{AI}+\mathrm{T}$ treatments was not observed (Fig. 4B). It is worth noting that the phenotype of ovary induced by $\mathrm{E}_{2}$ was slightly different from the ovary induced by the FPT, smaller in size with less evidence of primordial germ cells as noted in several turtle species (Merchant-Larios et al. 1997, Barske \& Capel 2010, Matsumoto \& Crews 2012). The phenotypic difference in testis, however, is less noticeable between the two triggers, the MPT and AI+T (Matsumoto \& Crews 2012). This observation may be attributed to a different pattern of landscape pathway during a mid period of development in the current study.

The experiment with steroid hormone antagonists revealed hormone-gene interactions, specifically a role of endogenous steroid hormones in the TSD system. Previous studies have shown that steroid hormone antagonists interrupt normal gonadal development, occasionally producing abnormal or intersex gonads in other species (Kang et al. 2006, Katoh et al. 2006). In our study, although steroid hormone antagonists resulted in little change in sex ratio and gonad morphology at hatching, the expression of candidate sex-determining genes has changed. For example, blocking endogenous $\mathrm{E}_{2}$ with a low dosage of $\mathrm{E}_{2}$ antagonist delayed the expression of aromatase at the FPT and a high dosage of $\mathrm{E}_{2}$ antagonist significantly suppressed aromatase (Fig. 5). This indicates that endogenous $E_{2}$ may be responsible for the manifestation of aromatase expression by a positive feedback mechanism at the FPT. Interestingly, despite the low levels of aromatase expression during development in the $\mathrm{E}_{2}$ antagonist-treated group, the ovaries at hatching did not exhibit any morphological or histological differences from control ovaries at FPT (data not shown). In reptiles and birds, aromatase is thought to be a key enzyme in determining the activation of male vs female pathways by balancing the production of estrogen (Elbrecht \& Smith 1992, Jeyasuria et al. 1994). Aromatase is still a candidate of the upstream factor to determine gonad sex in TSD species; however, our data suggest that the expression of aromatase may not be a sole determinant for an ovarian development. Similarly, the lack of an observable effect of the androgen antagonist flutamide on aromatase and Rspo1 gene expression at the MPT suggests that endogenous androgen may not be a primary factor for the suppression of typical ovarian markers at this temperature. Furthermore, Sox9 expression was not prominently affected by antagonist treatments either. These observations, along with hatching phenotypes, lead to the hypothesis that endogenous hormones may not participate in onset of the gene network of the sex-determining genes directed by temperature, but rather are involved in the gonad differentiation that follows. We cannot rule out the possibility that the dosage and timing of the application of antagonist in this study may not have been sufficient to completely block the endogenous steroid hormones. A previous study shows that blocking endogenous $E_{2}$ with multiple treatments of ICI 182780 leads to a delay in Sox9 downregulation in slider gonad at the FPT (Barske \& Capel 2010), which suggests that

Published by Bioscientifica Ltd. 
endogenous $\mathrm{E}_{2}$ has, at least to some extent, a suppressive effect on Sox9 expression.

We extended our investigations to elucidate whether the extraembryonic environment was involved in ligandinduced patterns of gene expression. Previous studies have shown that isolated embryonic gonads had the ability to sense and respond to the ambient temperature in TSD species (Moreno-Mendoza et al. 2001, Pieau \& Dorizzi 2004, Shoemaker-Daly et al. 2010). In the current study, the isolated gonads also exhibited the expression pattern of the candidate sex-determining genes similar to the one observed in ovo when treated with exogenous ligands and steroid hormone antagonists (Figs 6 and 7). However, the timing of the response of gene expression occurred late in the developmental stage with less intensity. For example, Rspo1 expression at FPT is not suppressed by AI $+\mathrm{T}$ treatment until 20 days after treatment (Fig. 6). In $\mathrm{E}_{2}$-treated gonads, both aromatase and Rspo1 expression increased, but with less intensity than the increase observed in ovo. One possible explanation is that isolated gonads lack the supporting cells normally migrated from underlying mesonephric tissue, resulting in a slow development and a lack of a morphological structure (Yao et al. 2004, Shoemaker-Daly et al. 2010). The antagonist treatments in vitro also followed the observation found in ovo: the $\mathrm{E}_{2}$ antagonist ICI 182780 suppressed the ovarian markers at the FPT, whereas the androgen antagonist flutamide lacked the consistent effect on the expression of these genes at the MPT (Fig. 7). It confirms the speculation in ovo that endogenous $\mathrm{E}_{2}$ positively affects ovarian markers at the FPT while endogenous androgen is not responsible for suppression of these genes at the MPT.

Overall, the current study shows that exogenous ligands modify the gonad phenotype by redirecting (suppress or activate) the expression of candidate sexdetermining genes. In vitro data further suggest that exogenous ligands, as wells as temperature, affect the expression of candidate sex-determining genes in gonads independently of other embryonic organs during development. Although the factors responsible for the regulation of these candidate sex-determining genes yet remain elusive, this study demonstrates further insights into the role of endogenous/exogenous steroid hormones in the process of sex determination in the TSD system.

\section{Supplementary data}

This is linked to the online version of the paper at http://dx.doi.org/10.1530/ JME-12-0260.

http://jme.endocrinology-journals.org DOI: 10.1530/JME-12-0260
(C) 2013 Society for Endocrinology Printed in Great Britain

\section{Declaration of interest}

The authors declare that there is no conflict of interest that could be perceived as prejudicing the impartiality of the research reported.

\section{Funding}

This work was supported by the National Science Foundation (grant number IOS-1051623).

\section{Author contribution statement}

$\mathrm{Y} M$ designed and performed the study. $\mathrm{R} Y$ and $\mathrm{C} T$ assisted animal dissection and organ cultures. D C contributed to the research design and supervised the project and $\mathrm{Y} M$ wrote the manuscript.

\section{Acknowledgements}

The authors are grateful to R Gillette and C Shoemaker-Daly for their helpful advice and $\mathrm{K}$ Kushiro for comments on the manuscript.

\section{References}

Barrionuevo F, Bagheri-Fam S, Klattig J, Kist R, Taketo MM, Englert C \& Scherer G 2006 Homozygous inactivation of Sox9 causes complete XY sex reversal in mice. Biology of Reproduction 74 195-201. (doi:10.1095/ biolreprod.105.045930)

Barske LA \& Capel B 2010 Sex determination: an avian sexual revolution. Nature 464 171-172. (doi:10.1038/464171a)

Bhattacharyya RS, Krishnan AV, Swami S \& Feldman D 2006 Fulvestrant (ICI 182 780) down-regulates androgen receptor expression and diminishes androgenic responses in LNCaP human prostate cancer cells. Molecular Cancer Therapeutics 5 1539-1549. (doi:10.1158/ 1535-7163.MCT-06-0065)

Bull JJ \& Vogt RC 1981 Temperature-sensitive periods of sex determination in Emydid turtles. Journal of Experimental Zoology 218 435-440. (doi:10.1002/jez.1402180315)

Chassot A-A, Ranc F, Gregoire EP, Roepers-Gajadien HL, Taketo MM, Camerino G, De Rooij DG, Schedl A \& Chaboissier M-C 2008 Activation of $\beta$-catenin signaling by Rspo1 controls differentiation of the mammalian ovary. Human Molecular Genetics 17 1264-1277. (doi:10.1093/hmg/ddn016)

Couse JF, Hewitt SC, Bunch DO, Sar M, Walker VR, Davis BJ \& Korach KS 1999 Postnatal sex reversal of the ovaries in mice lacking estrogen receptors $\alpha$ and $\beta$. Science 286 2328-2331. (doi:10.1126/science. 286.5448.2328)

Crews D 1996 Temperature-dependent sex determination: the interplay of steroid hormones and temperature. Zoological Science 13 1-13. (doi:10.2108/zsj.13.1)

Crews D \& Bergeron JM 1994 Role of reductase and aromatase in sex determination in the red-eared slider (Trachemys scripta), a turtle with temperature-dependent sex determination. Journal of Endocrinology 143 279-289. (doi:10.1677/joe.0.1430279)

Crews D, Cantú AR \& Bergeron JM 1996 Temperature and non-aromatizable androgens: a common pathway in male sex determination in a turtle with temperature-dependent sex determination? Journal of Endocrinology 149 457-463. (doi:10.1677/joe.0.1490457)

Dupont S, Dennefeld C, Krust A, Chambon P \& Mark M 2003 Expression of Sox9 in granulosa cells lacking the estrogen receptors, ER $\alpha$ and ER $\beta$. Developmental Dynamics 226 103-106. (doi:10.1002/dvdy.10202) 
Elbrecht A \& Smith RG 1992 Aromatase enzyme activity and sex determination in chickens. Science 255 467-470. (doi:10.1126/ science.1734525)

Greenbaum E 2002 A standardized series of embryonic stages for the emydid turtle Trachemys scripta. Canadian Journal of Zoology $\mathbf{8 0}$ 1350-1370. (doi:10.1139/z02-111)

Guiguen Y, Fostier A, Piferrer F \& Chang C-F 2010 Ovarian aromatase and estrogens: a pivotal role for gonadal sex differentiation and sex change in fish. General and Comparative Endocrinology 165 352-366. (doi:10.1016/j.ygcen.2009.03.002)

Huang B, Wang S, Ning Y, Lamb AN \& Bartley J 1999 Autosomal XX sex reversal caused by duplication of Sox9. American Journal of Medical Genetics 87 349-353. (doi:10.1002/(SICI)1096-8628 (19991203)87:4<349::AID-AJMG13 > 3.0.CO;2-N)

Jeyasuria P, Roosenburg WM \& Place AR 1994 Role of P-450 aromatase in sex determination of the diamondback terrapin, Malaclemys terrapin. Journal of Experimental Zoology 270 95-111. (doi:10.1002/ jez.1402700111)

Jolly C, Katsiadaki I, Le Belle N, Mayer I \& Dufour S 2006 Development of a stickleback kidney cell culture assay for the screening of androgenic and anti-androgenic endocrine disrupters. Aquatic Toxicology 79 158-166. (doi:10.1016/j.aquatox.2006.06.005)

Kang IJ, Hano T, Oshima Y, Yokota H, Tsuruda Y, Shimasaki Y \& Honjo T 2006 Anti-androgen flutamide affects gonadal development and reproduction in medaka (Oryzias latipes). Marine Environmental Research 62 (Suppl) S253-S257. (doi:10.1016/j.marenvres.2006.04.065)

Katoh H, Ogino Y \& Yamada G 2006 Cloning and expression analysis of androgen receptor gene in chicken embryogenesis. FEBS Letters 580 1607-1615. (doi:10.1016/j.febslet.2006.01.093)

Kitano T, Takamune K, Nagahama Y \& Abe SI 2000 Aromatase inhibitor and $17 \alpha$-methyltestosterone cause sex-reversal from genetical females to phenotypic males and suppression of p450 aromatase gene expression in japanese flounder (Paralichthys olivaceus). Molecular Reproduction and Development 56 1-5. (doi:10.1002/(SICI)1098-2795 (200005) 56:1<1::AID-MRD1 > 3.0.CO;2-3)

La Sala G, Farini D \& De Felici M 2010 Rapid estrogen signalling in mouse primordial germ cells. Experimental Cell Research 316 1716-1727. (doi:10.1016/j.yexcr.2010.03.024)

Matsumoto Y \& Crews D 2012 Molecular mechanisms of temperaturedependent sex determination in the context of ecological developmental biology. Molecular and Cellular Endocrinology 354 103-110. (doi:10.1016/j.mce.2011.10.012)

Merchant-Larios H, Ruiz-Ramirez S, Moreno-Mendoza N \& Marmolejo-Valencia A 1997 Correlation among thermosensitive period, estradiol response, and gonad differentiation in the sea turtle Lepidochelys olivacea. General and Comparative Endocrinology 107 373-385. (doi:10.1006/gcen.1997.6946)

Moreno-Mendoza N, Harley VR \& Merchant-Larios H 2001 Temperature regulates SOX9 expression in cultured gonads of Lepidochelys olivacea a species with temperature sex determination. Developmental Biology 229 319-326. (doi:10.1006/dbio.2000.9952)

Parma P, Radi O, Vidal V, Chaboissier MC, Dellambra E, Valentini S, Guerra L, Schedl A \& Camerino G 2006 R-spondin1 is essential in sex determination, skin differentiation and malignancy. Nature Genetics 38 1304-1309. (doi:10.1038/ng1907)

Pieau C \& Dorizzi M 2004 Oestrogens and temperature-dependent sex determination in reptiles: all is in the gonads. Journal of Endocrinology 181 367-377. (doi:10.1677/joe.0.1810367)
Ramsey M, Shoemaker C \& Crews D 2007 Gonadal expression of Sf1 and aromatase during sex determination in the red-eared slider turtle (Trachemys scripta), a reptile with temperature-dependent sex determination. Differentiation; Research in Biological Diversity 75 978-991. (doi:10.1111/j.1432-0436.2007.00182.x)

Scarpino S, Gillette R \& Crews D 2013 Multidimbio: an R package for the functional landscape analysis of multivariate data. Journal of Statistical Software (in press).

Schaeffer E, Marchionni L, Huang Z, Simons B, Blackman A, Yu W, Parmigiani G \& Berman D 2008 Androgen-induced programs for prostate epithelial growth and invasion arise in embryogenesis and are reactivated in cancer. Oncogene 27 7180-7191. (doi:10.1038/ onc.2008.327)

Shoemaker C, Ramsey M, Queen J \& Crews D 2007a Expression of Sox9, Mis, and Dmrt1 in the gonad of a species with temperature-dependent sex determination. Developmental Dynamics 236 1055-1063. (doi:10.1002/dvdy.21096)

Shoemaker CM, Queen J \& Crews D 2007b Response of candidate sexdetermining genes to changes in temperature reveals their involvement in the molecular network underlying temperature-dependent sex determination. Molecular Endocrinology 21 2750-2763. (doi:10.1210/ me.2007-0263)

Shoemaker-Daly CM, Jackson K, Yatsu R, Matsumoto Y \& Crews D 2010 Genetic network underlying temperature-dependent sex determination is endogenously regulated by temperature in isolated cultured Trachemys scripta gonads. Developmental Dynamics 239 1061-1075. (doi:10.1002/dvdy.22266)

Smith CA, Shoemaker CM, Roeszler KN, Queen J, Crews D \& Sinclair AH 2008 Cloning and expression of R-spondin 1 in different vertebrates suggests a conserved role in ovarian development. BMC Developmental Biology 8 72. (doi:10.1186/1471-213X-8-72)

Tomizuka K, Horikoshi K, Kitada R, Sugawara Y, Iba Y, Kojima A, Yoshitome A, Yamawaki K, Amagai M, Inoue A et al. 2008 R-spondin1 plays an essential role in ovarian development through positively regulating Wnt-4 signaling. Human Molecular Genetics 17 1278-1291. (doi:10.1093/hmg/ddn036)

Wibbels T \& Crews D 1994 Putative aromatase inhibitor induces male sex determination in a female unisexual lizard and in a turtle with temperature-dependent sex determination. Journal of Endocrinology 141 295-299. (doi:10.1677/joe.0.1410295)

Wibbels T, Bull JJ \& Crews D 1991 Chronology and morphology of temperature-dependent sex determination. Journal of Experimental Zoology 260 371-381. (doi:10.1002/jez.1402600311)

Wu YJ, Chen DW, Liu JL, Zhang JH, Luo HS \& Cui S 2009 Estradiol promotes pituitary cell proliferation and gonadotroph differentiation at different doses and with different mechanisms in chick embryo. Steroids 74 441-448. (doi:10.1016/j.steroids.2008.12.011)

Yano S, Tanaka M \& Nakao K 1995 Anti-tumour effect of aromatase inhibitor, CGS16949A, on human breast cancer cells. European Journal of Pharmacology 289 217-222. (doi:10.1016/09224106(95)90097-7)

Yao HH-C, DiNapoli L \& Capel B 2004 Cellular mechanisms of sex determination in the red-eared slider turtle, Trachemys scripta. Mechanisms of Development 121 1393-1401. (doi:10.1016/j.mod. 2004.06.001)

Yilmaz MB, Wolfe A, Zhao H, Brooks DC \& Bulun SE 2011 Aromatase promoter I.f is regulated by progesterone receptor in mouse hypothalamic neuronal cell lines. Journal of Molecular Endocrinology 47 69-80. (doi:10.1530/JME-10-0149)

Received in final form 22 March 2013

Accepted 26 March 2013

Accepted Preprint published online 26 March 2013 http://jme.endocrinology-journals.org DOI: 10.1530/JME-12-0260
() 2013 Society for Endocrinology Printed in Great Britain 